\title{
MCC950 Ameliorates Acute Liver Injury Through Modulating Macrophage Polarization and Myeloid-Derived Suppressor Cells Function
}

\author{
Wei Yan ${ }^{1,2}$, Yingchun Shen ${ }^{3}$, Jinny Huang ${ }^{2}$, Ling Lu ${ }^{1 *}$ and Qian Zhang ${ }^{4 *}$ \\ ${ }^{1}$ Hepatobiliary Center, The First Affiliated Hospital, Nanjing Medical University, Nanjing, China, ${ }^{2}$ Department of Surgery, \\ School of Medicine, Johns Hopkins University, Baltimore, MD, United States, ${ }^{3}$ Division of Allergy and Clinical Immunology, \\ School of Medicine, Johns Hopkins University, Baltimore, MD, United States, ${ }^{4}$ Department of General Surgery, The First \\ Affiliated Hospital of Nanjing Medical University, Nanjing, China
}

OPEN ACCESS

Edited by:

Hyeong-Geug Kim Indiana University Bloomington

United States

Reviewed by:

Changmin Peng,

George Washington University,

United States

Yu Qian,

University of Texas MD Anderson

Cancer Center, United States

${ }^{*}$ Correspondence:

Qian Zhang

zqsummer1031@njmu.edu.cn

Ling Lu

IVling@njmu.edu.cn

Specialty section:

This article was submitted to

Translational Medicine,

a section of the journal

Frontiers in Medicine

Received: 02 August 2021 Accepted: 15 October 2021 Published: 19 November 2021

Citation:

Yan W, Shen Y, Huang J, Lu L and Zhang Q (2021) MCC950 Ameliorates

Acute Liver Injury Through Modulating Macrophage Polarization and Myeloid-Derived Suppressor Cells Function. Front. Med. 8:752223. doi: 10.3389/fmed.2021.752223
Acute liver injury (ALI) raises high mortality rates due to a rapid pathological process. MCC950, a highly selective nod-like receptor family pyrin domain containing 3 (NLRP3) inhibitor, has already been reported to show strong hepatoprotective effects in many different liver diseases. In this study, we unveiled the role of MCC950 in carbon tetrachloride $\left(\mathrm{CCl}_{4}\right)$-induced $\mathrm{ALI}$ and its underlying molecular mechanisms on days 1 , 2, and 3. MCC950 could significantly inhibit liver injury, evidenced by decreased serum alamine aminotransferase (ALT) and aspartate aminotransferase (AST) levels on days 1 and 2, increased Albumin (ALB) level on day 3, and decreased histological score during the whole period. Moreover, lower M1 macrophage related to pro-inflammatory genes expression was observed in MCC950-treated ALI mice on day 1, while MCC950 pretreatment also polarized macrophage to $\mathrm{M} 2$ phenotype indicating anti-inflammatory response on days 2 and 3. Additionally, MDSC was significantly increased in blood, liver, and spleen in ALI mice at different time courses. Specifically, upregulated myeloid-derived suppressor cell (MDSC) proportions were found in blood and spleen on days 1 and 2, but showed decreased trend on day 3. However, liver MDSC numbers were increased on days 2 and 3, but no significance on day 1. In conclusion, MCC950 pretreatment alleviates $\mathrm{CCl}_{4}$-induced ALI through enhanced M2 macrophage and MDSC function at different time points of ALI. Further understanding of MCC950 in ALI may be a new potential therapeutic strategy.

Keywords: MCC950, acute liver injury, macrophage polarization, myeloid-derived suppressor cells, treatment

\section{INTRODUCTION}

Acute liver injury (ALI) has a rapid pathological process and is associated with a high mortality rate. It is already well-known that liver injury can be triggered by toxic chemicals, viruses, autoimmune diseases, and other factors, but there are currently no effective treatments (1). Therefore, it is necessary to investigate novel methods and drugs that can be used to treat the damage caused 
by acute liver injury. Carbon tetrachloride $\left(\mathrm{CCl}_{4}\right)$, oxidized by cytochrome P450 2E1 (CYP2E1) to generate highly reactive free radical trichloromethyl radical $\left(\cdot \mathrm{CCl}_{3}\right)$ and trichloromethyl peroxy radical $\left(\cdot \mathrm{OOCCl}_{3}\right)$ in the liver, has been widely used to construct the liver injury models both in vivo and in vitro $(2,3)$.

The pathogenesis mechanism for ALI contains a series of complicate processes such as inflammation, oxidative stress, and autophagy $(4,5)$. Among them, inflammation is the most common trigger for ALI (6). Among many known inflammatory cell complexes, the nod-like receptor (NLR) family pyrin domain containing 3 (NLRP3) inflammasome activation, which is composed of NLRP3, adaptor apoptosis-associated specklike protein containing a caspase recruitment domain (ASC), caspase-1, interleukin-1 $\beta$ (IL-1 $\beta$ ), and interleukin-18 (IL-18), is a well-characterized inflammatory factor in development of ALI (7-9). Hence, targeting on inhibiting NLRP3 inflammasome and investigating potential mechanism may be a crucial and effective aspect in liver injury. MCC950 is one of the most potent and selective NLRP3 inhibitors discovered to date and it can bind directly and specifically to NLRP3, irrespective of its activation state (10). More recently, MCC950 was reported to alleviate chronic cholestatic liver injury (11), fulminant hepatitis (12), and liver fibrosis (13). However, little is known about the role of MCC950 treatment in $\mathrm{CCl}_{4}$-induced acute liver injury.

The myeloid-derived suppressor cell (MDSC) population consists of a variety of heterogeneous immature myeloid cells and is a significant component of the immunosuppressive network (14). The therapeutic role of MDSCs in many different immune diseases such as liver failure and cancer has been explored due to their important role in immune suppression. Recently, it was discovered that in Acetaminophen (APAP)induced liver failure, Tumor Necrosis Factor Alpha (TNF$\alpha$ )/LipoPolySaccharide (LPS) MDSCs served a protective role by reducing intrahepatic infiltration of activated neutrophils (15). Additionally, in melanoma cells, NLRP3 activation can induce the expansion and immune evasion of MDSCs (16). Currently, there is no study on the role of MDSCs and MCC950 in ALI.

In liver diseases, the M2 macrophage participates in tissue repair and resolution of inflammation, whereas the M1 phenotype results in pro-inflammatory signaling based on their functions, secreted cytokines, and transcriptional profiles (17, 18). Moreover, inhibiting NLRP3-mediated M1 macrophage polarization in non-alcoholic steatohepatitis can lead to reduced liver steatosis and inflammation (19). However, the relationship between MCC950 and macrophage polarization in ALI still remains unknown.

In this study, we determined the effect of MCC950 treatment on $\mathrm{CCl}_{4}$-induced liver injury in a murine model. We first proved that MCC950 can alleviate $\mathrm{CCl}_{4}$-induced liver damage and we further provided evidence for the mechanism of protective effect of MCC950 against liver inflammation-MCC950 promotes M2 macrophage polarization and enhances MDSC function. All these data highlight the clinical potential of MCC950 as a treatment strategy for ALI.

\section{MATERIALS AND METHODS}

\section{Animals and Experimental Design}

All the procedures involving mice were performed in accordance with the approved protocols from the Animal Care and Use Committee of the Johns Hopkins University School of Medicine. An 8-week-old male C57BL/6 mice were used to construct ALI mouse model by $\mathrm{CCl}_{4}$ (Sigma, 270652, MO, USA) dissolved in olive oil $(1 \mathrm{mg} / \mathrm{kg}$ ) via intraperitoneal injection. MCC950 (Cell Signaling Technology, 86428S, MA, USA) was dissolved in sterile water and injected $(10 \mathrm{mg} / \mathrm{kg}) 1 \mathrm{~h}$ before $\mathrm{CCl}_{4}$ induction through intraperitoneal injection. Mouse was sacrificed and serum, blood, spleen, and liver tissues were collected for further detection on days 1,2 , and 3 .

\section{Histopathology and Immunofluorescence (IF)}

The 4- $\mu \mathrm{m}$ liver paraffin sections were stained with H\&E (Sigma, $\mathrm{MO}$, USA) according to the instructions of the manufacturer and images were taken under light microscope (Nikon, Tokyo, Japan). Additionally, for IF staining, $4 \mu \mathrm{m}$ liver frozen sections were fixed by paraformaldehyde for $10 \mathrm{~min}$ and washed with Tris-buffered saline with Tween (TBST) for three times and then blocked with $10 \%$ Fetal Bovine Serum (FBS) in TBST for $1 \mathrm{~h}$ at room temperature. The sections were then incubated with the primary antibodies against CD68 (1:100, BioRad, MCA1957, CA, USA) and Arg-1 (1:400, Proteintech, 16001-1, IL, USA) or isotype overnight at $4^{\circ} \mathrm{C}$. The sections were washed with TBST and then incubated with fluorescent dye-conjugated secondary antibodies for $60 \mathrm{~min}$ at room temperature. The fluorescent-positive cells were evaluated by using ImageJ Software version $1.50 \mathrm{e}$ National Institutes of Health, USA. Four to six sections from each sample were used for analysis.

\section{Ribonucleic Acid Extraction, Reverse Transcription, and Quantitative Real-Time PCR (RT-PCR)}

Total RNA of liver tissues from different groups was extracted with the RNeasy Plus Mini Kit (QIAGEN, Hilden,

TABLE 1 | Sequences of target genes for real-time PCR.

\begin{tabular}{|c|c|c|}
\hline Gene & Forward primer $\left(5^{\prime}\right.$ to $\left.3^{\prime}\right)$ & Reverse primer $\left(5^{\prime}\right.$ to $\left.3^{\prime}\right)$ \\
\hline $\begin{array}{l}\beta \text {-actin } \\
\text { (mouse) }\end{array}$ & GGTTGTCTCCTGCGACTTCA & TGGTCCAGGGTTTCTACTCC \\
\hline $\begin{array}{l}\text { NLRP3 } \\
\text { (mouse) }\end{array}$ & ATTACCCGCCCGAGAAAGG & TCGCAGCAAAGATCCACACAG \\
\hline $\begin{array}{l}\text { IL-1 } \beta \\
\text { (mouse) }\end{array}$ & GCAACTGTTCCTGAACTCAACT & ATCTITGGGGTCCGTCAACT \\
\hline $\begin{array}{l}\text { IL-6 } \\
\text { (mouse) }\end{array}$ & TAGTCCTTCCTACCCCAATITCC & TTGGTCCTTAGCCACTCCTTC \\
\hline $\begin{array}{l}\text { iNOS } \\
\text { (mouse) }\end{array}$ & GTTCTCAGCCCAACAATACAAGA & GTGGACGGGTCGATGTCAC \\
\hline $\begin{array}{l}\text { Arg-1 } \\
\text { (mouse) }\end{array}$ & CTCCAAGCCAAAGTCCTTAGAG & AGGAGCTGTCATTAGGGACATC \\
\hline $\begin{array}{l}\text { Ym1/2 } \\
\text { (mouse) }\end{array}$ & CAGGTCTGGCAATTCTTCTGAA & GTCTTGCTCATGTGTGTAAGTGA \\
\hline $\begin{array}{l}\text { Fizz1 } \\
\text { (mouse) }\end{array}$ & CCAATCCAGCTAACTATCССТCC & ACCCAGTAGCAGTCATCCCA \\
\hline
\end{tabular}


Germany) according to the instructions of the manufacturer. Complementary DNA (cDNA) was synthesized through the High-Capacity RNA-to-cDNA Kit (Applied Biosystems, MA, USA). Quantitative RT-PCR was performed by the SYBR Green PCR Master Mix (Applied Biosystems, MA, USA) by using the 7300 Real-Time PCR System (Applied Biosystems, MA, USA). Quantitation of the relative expression levels of each gene was detected in triplicate and calculated by using the $2^{-\Delta \Delta C T}$ method. $\beta$-actin was used as an endogenous control. The primers used are provided in Table 1.

\section{Western Blotting (WB)}

For WB analysis, an equal amount of total protein $(20-50 \mu \mathrm{g})$ was loaded onto a $12 \%$ Tris-Glycine Gel in the NuPAGE 2morpholino-ethanesulfonic acid (MES) sodium dodecyl sulfate (SDS) Running Buffer (Thermo Fisher Scientific, MA, USA) and then transferred by using the iBlot 2 NC Stack System (Thermo Fisher Scientific, MA, USA). The membranes were blocked in 5\% non-fat milk in TBST for $1 \mathrm{~h}$ at room temperature and probed with primary antibodies (NLRP3, 1:1,000; Novus Biologicals, 12,446, CO, USA; IL- $1 \beta, 1: 1,000$; R\&D Systems, AF$401, \mathrm{MN}, \mathrm{USA})$ overnight at $4^{\circ} \mathrm{C}$. Species appropriate secondary antibodies conjugated to IRDye 680RD were used according to the instructions of the manufacturer. Band intensities were quantified by the Image J Software and quantification on each band was normalized to glyceraldehyde 3-phosphate dehydrogenase (GAPDH).

\section{Preparation of the Cell Suspension}

After mice were anesthetized, blood was collected via central vein, Ammonium-Chloride-Potassium (ACK) lysis buffer was used to lyse red blood cells (RBCs), and then wash with phosphate-buffered saline (PBS). During dissection, spleens were removed and collected in RPMI-1640 solution (11875093, Thermo Fisher Scientific, MA, USA) on the ice. Single cells were obtained after mashing the spleen through a $70-\mu$ m nylon cell strainer (VWR International, PA, USA) followed by $10 \mathrm{~min}$ treatment with $5 \mathrm{ml} \mathrm{RBC}$ lysis buffer (420302, BioLegend, CA, USA) at room temperature. After washing with RPMI-1640 solution, cells were resuspended in RPMI-1640 + 2\% FBS. Livers were collected and digested in $1 \mathrm{mg} / \mathrm{ml}$ collagenase II for $30 \mathrm{~min}$ in $37^{\circ} \mathrm{C}$ followed by being mashed through a $70-\mu \mathrm{m}$ strainer to obtain the cell suspension containing hepatocytes and non-parenchymal cells (NPCs). The hepatocytes were removed

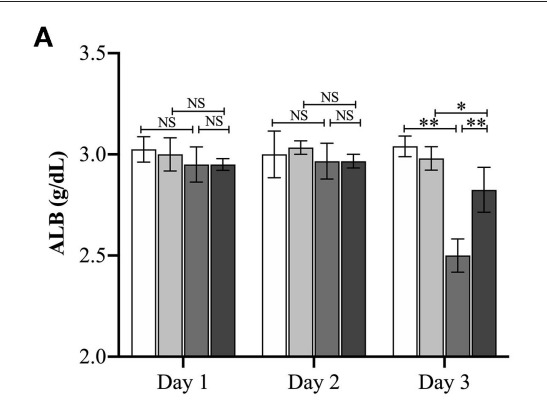

D

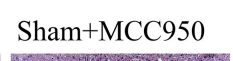

B
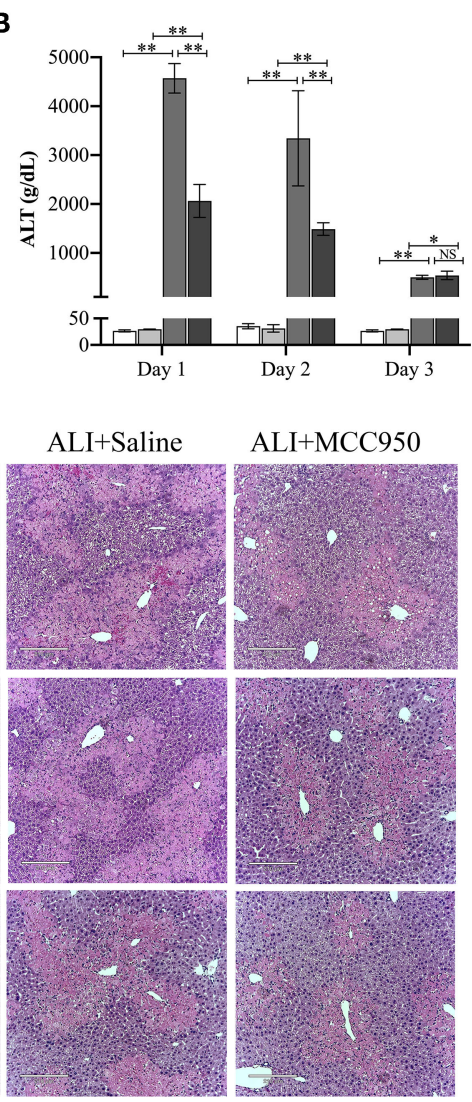

C

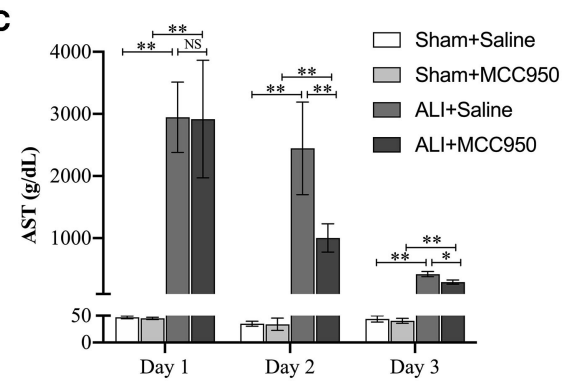

E

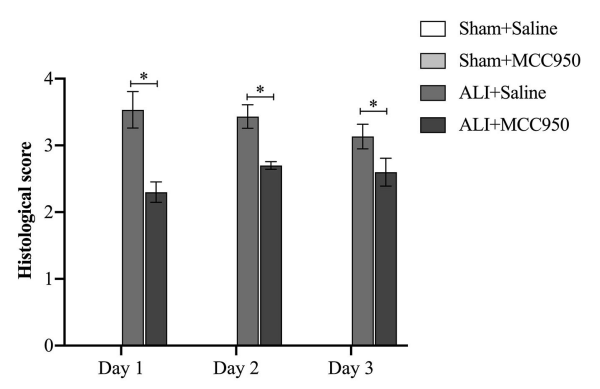

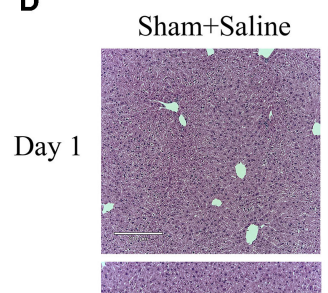

Day 3

Day 2
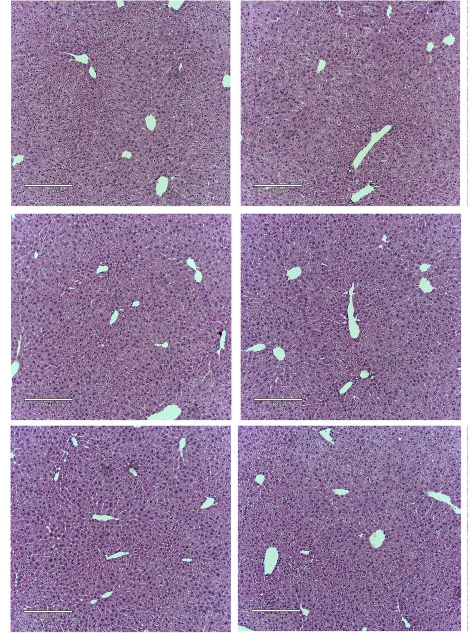

FIGURE 1 | MCC950 alleviates acute liver injury. (A) Time course of ALB serum levels in different mice ( $n=8)$. (B) Time course of ALT serum levels in different mice ( $n$ $=8)$. (C) Time course of AST serum levels in different mice $(n=8)$. (D) Histological examination of mouse paraffin liver sections stained with H\&E staining from carbon tetrachloride $\left(\mathrm{CCl}_{4}\right)$-treated mice pretreated with vehicle or MCC950. (E) The histological scores for liver sections in different groups. Data are presented as mean \pm SEM. NS: No significance. ${ }^{\star} p<0.05,{ }^{\star \star} p<0.01$. Intergroup differences are determined by the Student's $t$-test. 
by centrifugation at a speed of 50 Relative Centrifugal Force (RCF) for $5 \mathrm{~min}$ and then the NPCs were collected from the supernatant above after centrifugation at $400 \mathrm{rcf}$ for $5 \mathrm{~min}$. After 10 min of RBC lysis buffer, NPCs were suspended in RPMI-1640 solution.

\section{Flow Cytometry}

Single-cell suspensions $\left(2 \times 10^{5}\right)$ from blood, spleen, and liver were blocked with antimouse CD16/32 (1:100, BioLegend, 101302, CA, USA) diluted in PBS for $20 \mathrm{~min}$ and then stained with fluorescently-labeled antibodies against surface markers of MDSC (CD11b and Gr-1, 1:200, BioLegend, 101212 and 108408, $\mathrm{CA}$, USA) for $40 \mathrm{~min}$ at $4^{\circ} \mathrm{C}$. fluorescence-activated single cell sorting (FACS) analysis was performed on the FACSCalibur Flow Cytometer (BD Biosciences, CA, USA) by using the FlowJo Software (CA, USA).

\section{Statistical Analysis}

All the experimental data were analyzed by using the GraphPad Prism (CA, USA) and were presented as the means with error bars showing the SEM (mean \pm SEM). Analysis of differences was performed by using the two-tailed Student's $t$ test or with the ANOVA. $P$-values $<0.05$ were considered as statistically significant.

\section{RESULTS}

\section{MCC950 Alleviates Acute Liver Injury}

To better understand the role of NLRP3 inflammasome in ALI, MCC950, a highly selective NLRP3 inhibitor, was used to treat animals $1 \mathrm{~h}$ before $\mathrm{CCl}_{4}$ injection. The biochemical markers of hepatocellular damage, serum ALB (Figure 1A), ALT (Figure 1B), and AST (Figure 1C) concentration levels showed that $\mathrm{CCl}_{4}$ injection can lead to liver damage at different time points, while the most severe damage was observed on day 1 . Interestingly, increased serum ALB level was observed on day 3 , but no changes on days 1 and 2 (Figure 1A). Moreover, MCC950 treatment significantly reduced AST (Figure 1B) and ALT (Figure 1C) levels, especially on days 1 and 2, while no significant reduction occurred on day 3. Meanwhile, $\mathrm{H} \& \mathrm{E}$ staining showed that MCC950 treatment attenuated liver injury with less necrosis and inflammatory cell infiltration around the blood vessels at all the time points (Figures 1D,E). Given all the evidence, MCC950 indeed alleviates $\mathrm{CCl}_{4}$-induced ALI.

\section{MCC950 Inhibits Liver NLRP3 Inflammasome Activation in ALI Mice}

As shown in Figure 2, the expression of NLRP3 and IL-1 $\beta$ in liver tissues was significantly increased in $\mathrm{CCl}_{4}$-induced ALI group compared with control group evaluated by WB (Figures 2A-D) and RT-PCR (Figure 2E) on days 1, 2, and 3. Moreover, MCC950 treatment markedly inhibited the expression of NLRP3 and IL-1 $\beta$ in ALI mice at different time points.

\section{MCC950 Ameliorates ALI via Enhanced MDSC Function}

Next, we continued to use flow cytometry to assess the role of MCC950 treatment on MDSC function. As shown in Figure 3A and Supplementary Figure 1, MDSC numbers were increased in spleen, blood, and liver of ALI group compared with control group and sham group. Moreover, MCC950 treatment can upregulate spleen and blood MDSC proportions in days 1 and 2 , but exist reduced tendency on day 3 (Figures 3B,C). However, liver MDSC numbers were increased on days 2 and 3, while no significance on day 1 (Figure 3D). Combine together, we proposed that MCC950 treatment can firstly increase spleen and blood MDSC on days 1 and 2 and then recruit MDSC into liver from days 2 to 3 during liver injury process.

\section{MCC950 Prevents ALI Through Polarizing Macrophage Into M2 Phenotype}

To further investigate whether or not MCC950 attenuates liver damage through macrophage polarization, M1 [inducible nitric oxide synthase (iNOS) and interleukin-6 (IL-6)] and M2 (Fizz1, Arg-1, and Ym1/2) phenotypes were evaluated by RT-PCR and IF staining. As shown in Figure 4A, M1-related genes such as iNOS and IL- 6 were reduced on days 1 and 2, but no significance on day 3. Additionally, all the M2-related genes were increased in ALI group and MCC950 treatment can lead to higher expression of Fizz1, Arg-1, and Ym1/2 on days 2 and 3, while no obvious significance on day 1 (Figure 4B). Moreover, we continued using IF costaining of CD68 and Arg-1 in the liver tissues. As shown in Figures 4C,D, double-positive cells were increased on days 2 and 3, while no changes on day 1 , which are consistent with RTPCR detection. Thus, these results prove that MCC950 attenuates ALI through polarizing macrophage into M2 phenotype on days 2 and 3.

\section{Cytokines Dysfunction Can Be Rescued by MCC950 Treatment in ALI}

Finally, cytokines (IL-1 $\beta$, IL-2, IL-6, IL-10, and TNF- $\alpha$ ) in serum were detected in different time points (Figure 5). MCC950 treatment can reduce IL- $1 \beta$, IL- 6 , and TNF- $\alpha(\mathrm{pg} / \mathrm{ml})$ level on days 1,2 , and 3 , while it can only reduce IL-6 ( $\mathrm{pg} / \mathrm{ml})$ level on days 1 and 3. Of note, MCC950 also can enhance IL-10 (pg/ml) expression on days 2 and 3. These data indicate that MCC $950 \mathrm{can}$ rescue cytokines dysfunction in ALI.

\section{DISCUSSION}

Acute massive or chronic persistent liver damages can result in liver failure. Developing an alternative therapeutic stratagem to reduce injury, prevent progression, and restore liver function is of significant clinical relevance. In this study, we provided convincing evidence that pretreatment with MCC950, a NLRP3specific inhibitor, effectively alleviates $\mathrm{CCl}_{4}$-induced ALI in a murine model.

Nowadays, inflammation is the most prevalent underlying pathology in ALI. It is well-documented that NLRP3 inflammasome plays a significant role in both the early and 
progressive inflammation $(20,21)$. Recently, several compounds have emerged as inhibitors for the NLRP3 inflammasome cascade (22); among all the inhibitors of NLRP3 inflammasome, MCC950 shows excellent potency and high target selectivity, yet its pharmacokinetic and toxicokinetic properties limited its therapeutic development in the clinical settings (10). Previous studies have demonstrated that MCC950 treatment could reduce IL- $1 \beta$ production and attenuate the severity of lung ischemia-reperfusion injury (23), ulcerative colitis (24), myocardial infarction (25), multiple sclerosis (22), and liver transplantation (26). Importantly, MCC950 exerts strong hepatoprotective properties in multiple types of mouse liver injury models. A recent study suggested that MCC950 exerts protective effects for liver inflammation and fibrosis in two models of Non-alcoholic steatohepatitis (NASH) (27). Also, in a bile duct ligation (BDL) model for cholestasis, MCC950 has been demonstrated to reduce liver fibrosis through inhibiting NLRP3 and the mechanism was partially attributed to inhibition of Toll-like receptor signaling (11). Additionally, MCC950 had also been reported to reduce liver inflammatory response and fibrosis of testosterone-treated mice (13). However, its efficacy in ALI remains unknown.

In this study, $\mathrm{CCl}_{4}$-induced ALI model was constructed with MCC950 or vehicle pretreatment. Mice were sacrificed during both the early phase (days 1 and 2) and also the late phase (day 3) in order to determine the mechanism of the treatment. Through detection of H\&E staining, serum ALB, AST and ALT levels, and NLRP3 inflammasome levels, we found that activated NLRP3 and IL-1 $\beta$ expressions are coincident with the severity of histopathological damage in the liver. Moreover, MCC950 treatment actually blocked NLRP3 and IL-1 $\beta$ expression at different time points. Interestingly, MCC950 treatment in ALI mice can reduce liver injury and function at all the different time points, especially in the early phase days 1 and 2, indicating MCC950 can be viewed as alternative therapeutic target in ALI.

Recently, MDSCs have been gaining increased attention due to its ability to reduce inflammation and limit tissue damage by modulating both the innate and adaptive immune responses $(28,29)$. In this study, we found that for ALI mice, the MDSC population increased in spleen, blood, and liver tissues in both the early phase and the late phase after $\mathrm{CCL}_{4}$ injection. To investigate how MCC950 treatment affected MDSC population, we also evaluated the MDSC numbers in MCC950treated mice at different time points. Notably, in the early phase, MCC950 treatment can increase MDSC numbers in spleen and blood, but not increase MDSC numbers in liver on day 1. Surprisingly, in the late phase (day 3), MCC950 can enhance MDSC number in liver, but reduced tendency in spleen and blood was observed. Accordingly, it is well-founded that enhanced MDSC numbers generated after MCC950 treatment can participate in rescuing process in the early phase and regeneration process in the late phase. However, the molecular mechanism through which driving MDSC mobilization into inflamed liver remains elusive. Upon NLRP3 activation, the inactive IL-1 $\beta$ precursor is processed by caspase- 1 to active, mature IL-1 $\beta$, which could induce cytokines associated with MDSC expansion such as IL-6 and IL-8 (30). A recent study
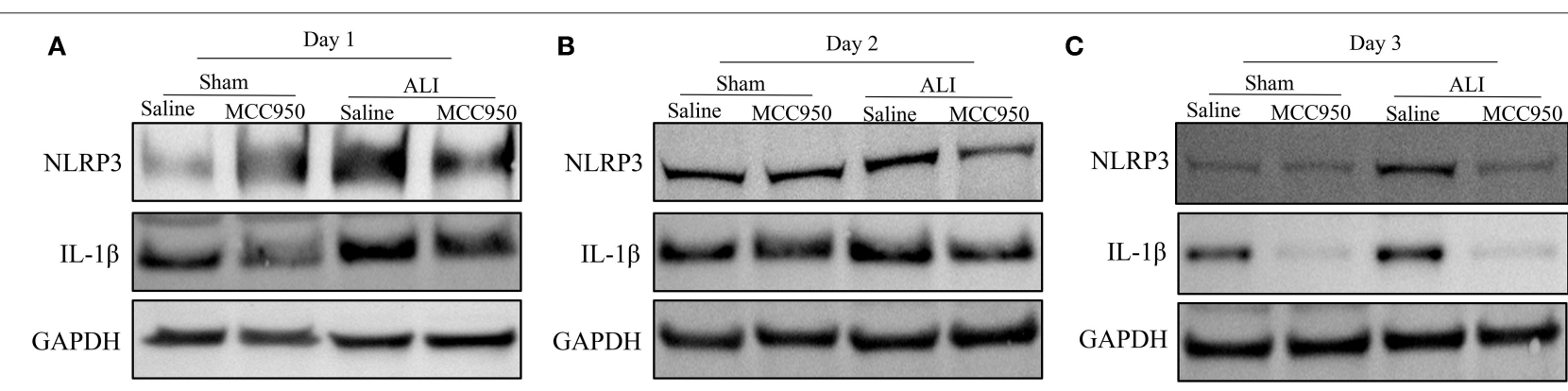

D

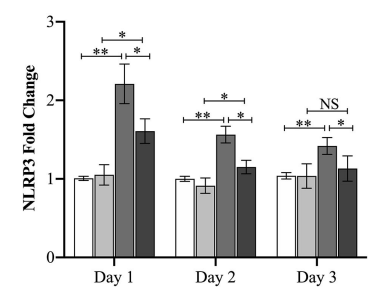

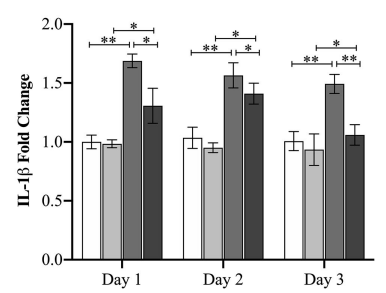

E
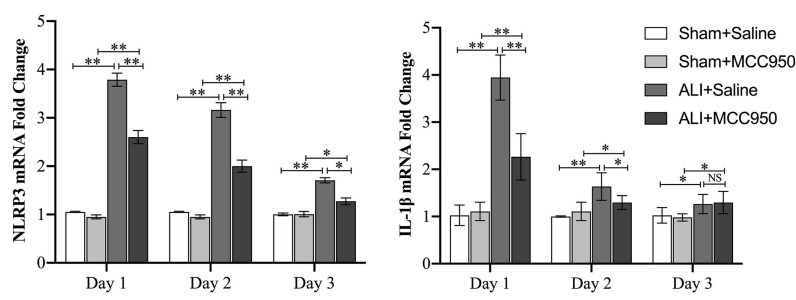

FIGURE 2 | The nod-like receptor family pyrin domain containing 3 (NLRP3) inflammasome activation in acute liver injury mice is inhibited by MCC950. Western blot

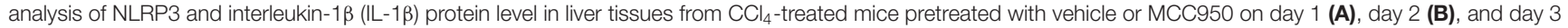
(C), GAPDH was detected as the loading control. (D) Quantitative analysis of western blots (A-C), ( $n=3)$. (E) Real-time PCR (RT-PCR) analysis of liver NLRP3 and IL-1 $\beta$ messenger RNA (mRNA) level in different mice. Data are presented as mean \pm SEM. NS: No significance. ${ }^{*} p<0.05,{ }^{\star \star} p<0.01$. Intergroup differences are determined by the Student's $t$-test. 


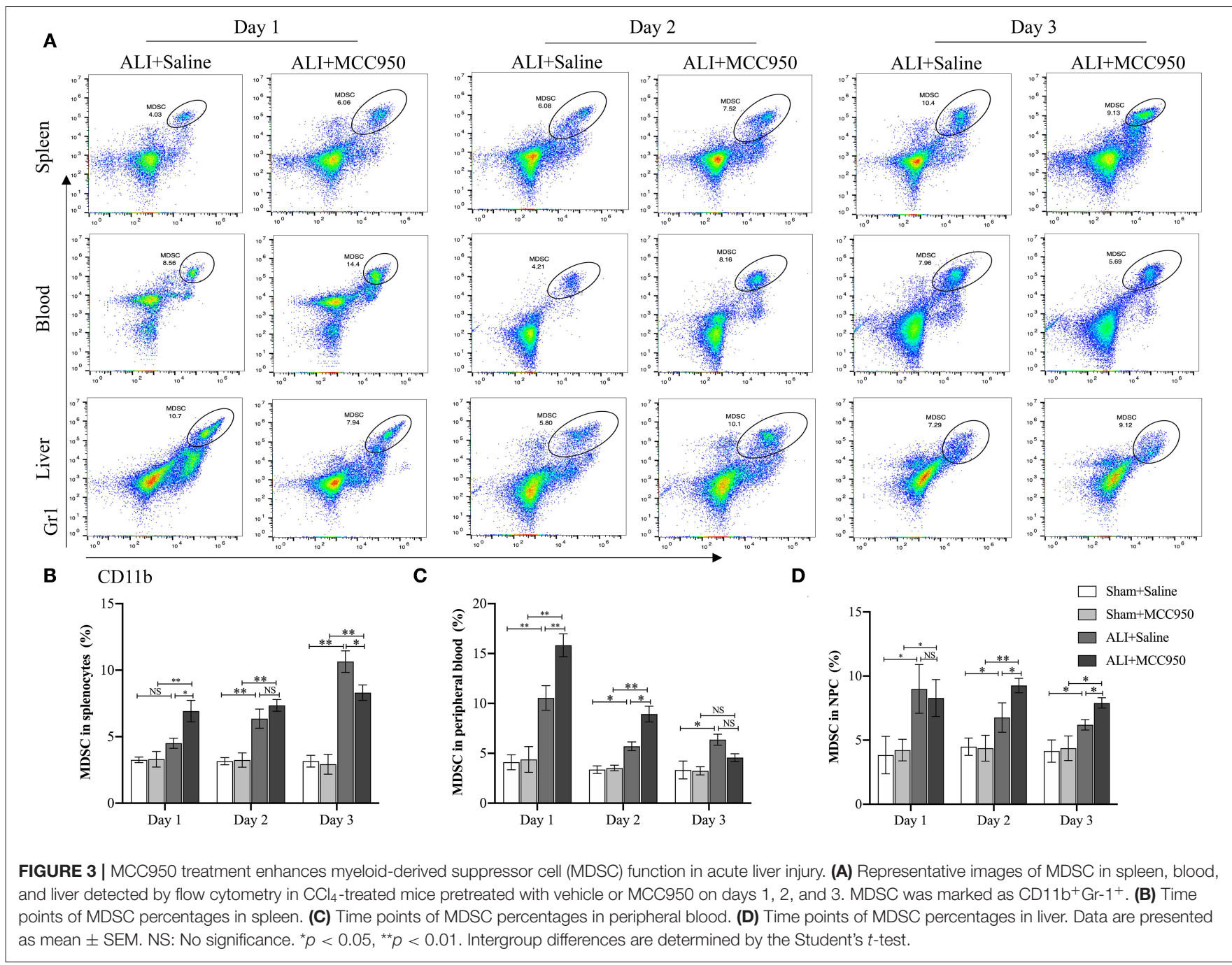

suggested that IL-25 is highly expressed in both the human and mouse liver and plays a critical function in maintaining the homeostasis and limiting local inflammation through recruiting MDSC (31). Another study further demonstrated that during the pathogenesis of Propionibacterium acnes/LPSinduced fulminant hepatitis (FH), a protein kinase $\mathrm{Tpl} 2$ could mediate the induction of MDSC-attracting chemokines such as CXC chemokine ligand-1 (CXCL1) and CXC chemokine ligand-2 (CXCL2) through modulating IL-25 signaling in hepatocytes, which could further promote the recruitment of MDSC into liver (32). Moreover, CCL17 was also reported to be a MDSC-attracting chemokine induced by IL-25 in Dgalactose (D-Gal)/LPS-induced fulminant hepatitis $(\mathrm{FH})$ mice (31). These results support the function of MDSCs in tissue protection in terms of inflammation and provide evidence that MCC950 could rescue liver damage via recruiting MDSC to liver.

Generally, M1 macrophages are thought to promote cytotoxic/pro-inflammatory factors such as IL-1 $\beta$, TNF- $\alpha$,
IL-6, and iNOS, which can cause cell apoptosis and tissue damage, whereas M2 macrophages are associated with tissue repair/reparative fibrosis via upregulating Fizz1, Arg-1, Ym1/2, and IL-10 (33-35). In addition, these M1 and M2 macrophages can modulate hepatic lesions induced by hepatotoxicants (36). Therefore, we decided to evaluate the effect of MCC950 on macrophage polarization in ALI. Using RT-PCR, we found that MCC950 treatment can upregulate M2-related genes (Fizz1, Arg-1, Ym1/2, and IL-10), but decrease M1-related genes (IL-1 $\beta$, TNF- $\alpha$, IL-6, and iNOS). Double IF analyses by CD68 and Arg- 1 further supported our analysis.

As mentioned above, MCC950 can influence cytokine levels. We measured the levels of IL-1 $\beta$, IL-2, IL-6, IL-10, and TNF$\alpha$ in serum. Consistent with pathological results, MCC950 can alleviate liver damage through reducing IL-1 $\beta$, IL-2, IL6 , and TNF- $\alpha$, but enhancing IL-10 production. In line with our data, many other studies reported that MCC950 could reduce the production of pro-inflammatory cytokines including IL-1 $\beta$, IL-18, IL-1 $\alpha$, IFN $\gamma$, TNF- $\alpha$, IL-6, IL-17, and 


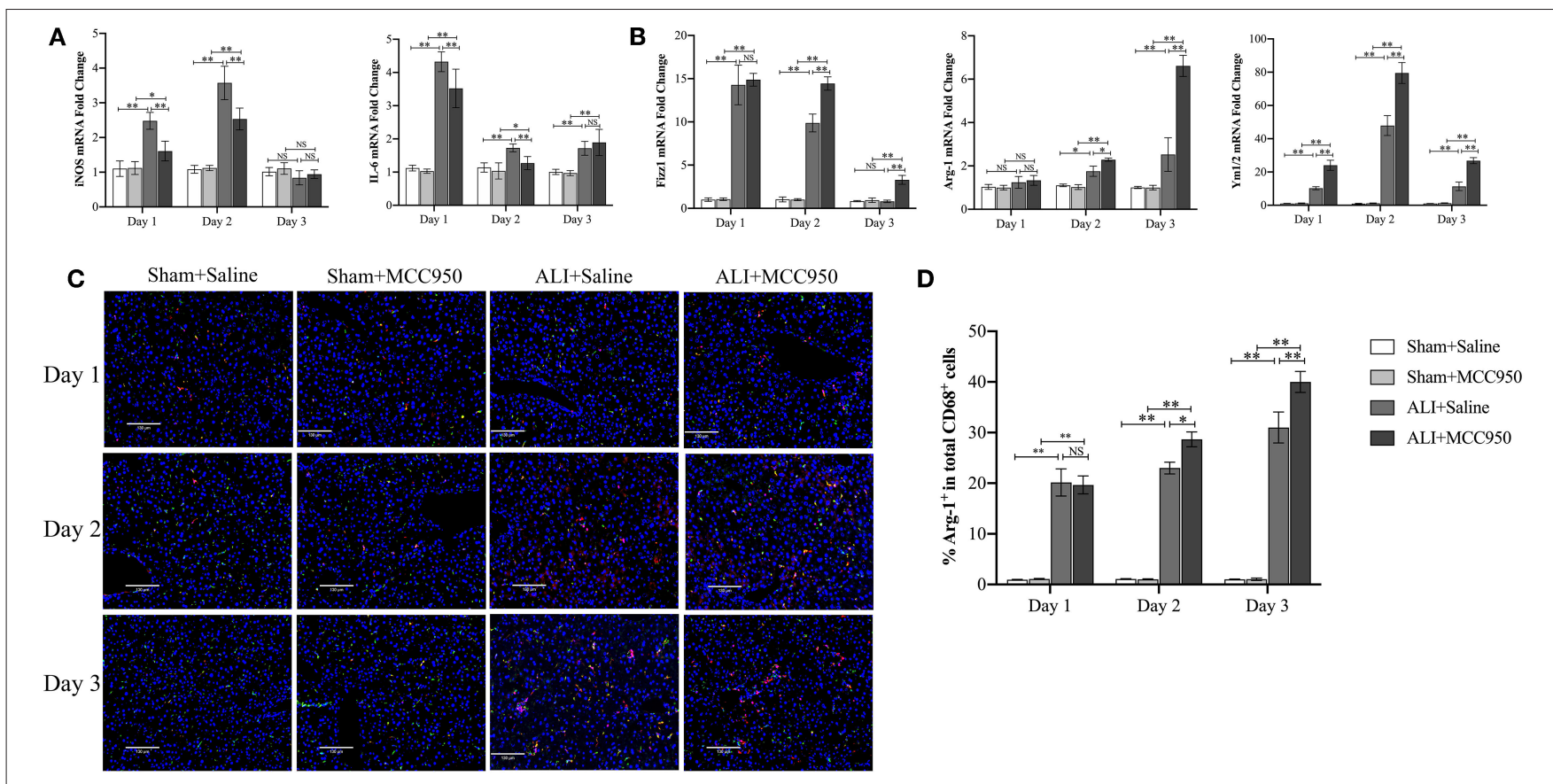

FIGURE 4 | MCC950 prevents acute liver injury through polarizing macrophage into M2 phenotype. (A) Real-time PCR (RT-PCR) analysis of M1-related genes such

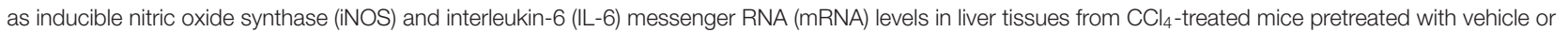
MCC950 on days 1, 2, and 3. (B) RT-PCR analysis of M2-related genes such as Fizz1, Arg-1, and Ym1/2 mRNA levels in liver tissues from CCl 4 -treated mice pretreated with vehicle or MCC950 on days 1, 2, and 3. (C) Representative images of double-immune fluorescence staining with CD68 and Arg-1 in frozen liver section from different mice. Nuclei were counterstained with 4'-6-diamidino-2-phenylindole dihydrochloride (DAPI). (D) Quantitation of liver tissue M2 macrophages as indicated by the percentage of $\mathrm{CD}^{+} 8^{+} \mathrm{Arg}-1^{+}(\% \mathrm{M} 2)$ among total CD68 ${ }^{+}$cells $\left(n=8-9\right.$ field/group). Data are presented as mean \pm SEM. NS: No significance. ${ }^{*} p<$ $0.05,{ }^{* *} p<0.01$. Intergroup differences are determined by the Student's $t$-test.

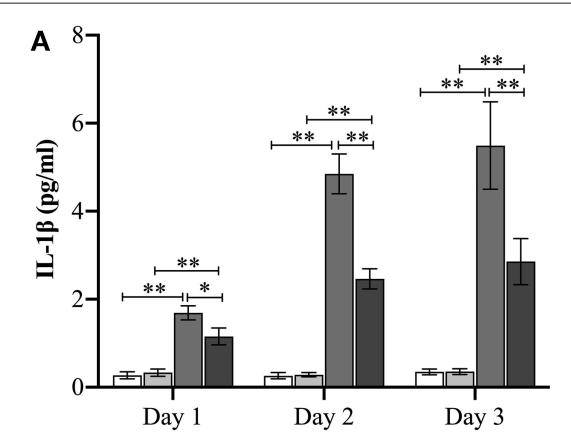

C

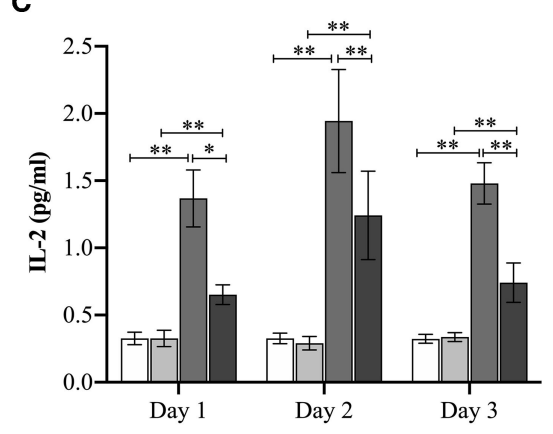

B

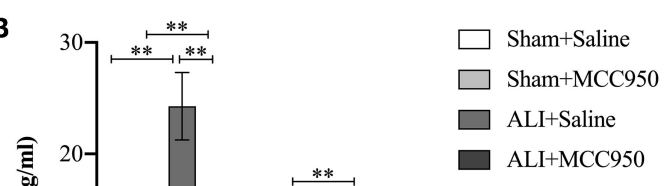

FIGURE 5 | MCC950 treatment rescues cytokines dysfunction in acute liver injury. Levels of IL-1 3 (A), TNF- $\alpha$ (B), IL-2 (C), IL-6 (D), and IL-10 (E) in serum from mice in different groups. Data are presented as mean \pm SEM. NS: No significance ${ }^{\star} p<0.05,{ }^{\star *} p<0.01$. Intergroup differences are determined by the Student's $t$-test. 
chemokine Macrophage Inflammatory Protein 1-? (MIP-1 $\alpha$ ) and inhibit neutrophil infiltration and cell apoptosis (37-39), which may be one of the reasons why MCC950 plays a role in suppressing inflammation and improving liver function in ALI.

In conclusion, this study demonstrated that MCC950 treatment in $\mathrm{CCl}_{4}$-induced ALI can recruit MDSCs, promote M2 macrophage polarization, and modulate cytokine levels by decreasing pro-inflammatory and increasing anti-inflammatory cytokines. These protective effects happen both during the early phase (days 1 and 2) and the late phase (day 3) post-injury. Due to its ability to suppress inflammation and improve liver function, MCC950 treatment has important protective effects in the progression of ALI and may lead to new therapeutic strategies for ALI.

\section{DATA AVAILABILITY STATEMENT}

The original contributions presented in the study are included in the article/Supplementary Material, further inquiries can be directed to the corresponding author/s.

\section{ETHICS STATEMENT}

All procedures involving mice were performed in accordance with the approved protocol from the Animal Care and Use Committee of the Johns Hopkins University School of Medicine (No. MO18M233).

\section{REFERENCES}

1. Asrani SK, Devarbhavi H, Eaton J, Kamath PS. Burden of liver diseases in the world. J Hepatol. (2019) 70:151-71. doi: 10.1016/j.jhep.2018. 09.014

2. Lyu H, Wang H, Li L, Zhu J, Chen F, Chen Y, et al. Hepatocytespecific deficiency of Nrf2 exacerbates carbon tetrachloride-induced liver fibrosis via aggravated hepatocyte injury and subsequent inflammatory and fibrogenic responses. Free Radic Biol Med. (2020) 150:136-47. doi: 10.1016/j.freeradbiomed.2020.02.015

3. Feng R, Wang M, Yan C, Li P, Chen M, He C, et al. Endogenous n-3 fatty acids alleviate carbon-tetrachloride-induced acute liver injury in fat-1 transgenic mice. Oxid Med Cell Longev. (2016) 2016:7962948. doi: 10.1155/2016/79 62948

4. Benjafield AV, Ayas NT, Eastwood PR, Heinzer R, Ip MSM, Morrell MJ, et al. Estimation of the global prevalence and burden of obstructive sleep apnoea: a literature-based analysis. Lancet Respir Med. (2019) 7:687-98. doi: 10.1016/S2213-2600(19)30198-5

5. Huang S, Mo C, Zeng T, Lai Y, Zhou C, Xie S, et al. Lupeol ameliorates LPS/D-GalN induced acute hepatic damage by suppressing inflammation and oxidative stress through TGFbetal-Nrf2 signal pathway. Aging. (2021) 13:6592-605. doi: 10.18632/aging.202409

6. Zhang LG, Chen J, Meng JL, Zhang Y, Liu Y, Zhan CS, et al. Effect of alcohol on chronic pelvic pain and prostatic inflammation in a mouse model of experimental autoimmune prostatitis. Prostate. (2019) 79:1439-49. doi: $10.1002 /$ pros. 23866

7. Wree A, Eguchi A, McGeough MD, Pena CA, Johnson CD, Canbay A, et al. NLRP3 inflammasome activation results in hepatocyte pyroptosis, liver inflammation, and fibrosis in mice. Hepatology. (2014) 59:898-910. doi: 10.1002/hep.26592

\section{AUTHOR CONTRIBUTIONS}

QZ and WY conceived and designed the study and finalized the manuscript. JH conducted the experiments, analyzed data, and edited the manuscript. LL and YS mainly edited the manuscript. All the authors read and approved the final version of the manuscript.

\section{FUNDING}

This study was supported by grants from the National Natural Science Foundation of China (81971495, 81571564, and 91442117), the Chinese Academy of Medical Sciences (CAMS) Innovation Fund for Medical Sciences (No. 2019-I2M5-035), and the National Science Foundation of Jiangsu Province (BRA2017533, BK20191490, and BE2016766).

\section{ACKNOWLEDGMENTS}

We thank the editors for careful review.

\section{SUPPLEMENTARY MATERIAL}

The Supplementary Material for this article can be found online at: https://www.frontiersin.org/articles/10.3389/fmed. 2021.752223/full\#supplementary-material

Supplementary Figure 1 | Representative images of MDSC in spleen, blood, and liver detected by flow cytometry in sham group pretreated with vehicle or MCC950 on days 1, 2, and 3. MDSC was marked as CD11 $\mathrm{b}^{+} \mathrm{Gr}-1^{+}$.
8. Zou J, Wang SP, Wang YT, Wan JB. Regulation of the NLRP3 inflammasome with natural products against chemical-induced liver injury. Pharmacol Res. (2021) 164:105388. doi: 10.1016/j.phrs.2020.105388

9. Wang L, Hauenstein AV. The NLRP3 inflammasome: mechanism of action, role in disease and therapies. Mol Aspects Med. (2020) 76:100889. doi: 10.1016/j.mam.2020.100889

10. Corcoran S E, Halai R, Cooper M A. Pharmacological inhibition of the nodlike receptor family pyrin domain containing 3 inflammasome with MCC950. Pharmacol Rev. (2021) 73:968-1000. doi: 10.1124/pharmrev.120.000171

11. Frissen M, Liao L, Schneider KM, Djudjaj S, Haybaeck J, Wree A, et al. Bidirectional role of NLRP3 during acute and chronic cholestatic liver injury. Hepatology. (2021) 73:1836-54. doi: 10.1002/hep.31494

12. Pourcet B, Zecchin M, Ferri L, Beauchamp J, Sitaula S, Billon C, et al. Nuclear receptor subfamily 1 group D member 1 regulates circadian activity of NLRP3 inflammasome to reduce the severity of fulminant hepatitis in mice. Gastroenterology. (2018) 154:1449-64. doi: 10.1053/j.gastro.2017.12.019

13. Ma X, Zhou Y, Qiao B, Jiang S, Shen Q, Han Y, et al. Androgen aggravates liver fibrosis by activation of NLRP3 inflammasome in CCl4-induced liver injury mouse model. Am J Physiol Endocrinol Metab. (2020) 318:E817-29. doi: 10.1152/ajpendo.00427.2019

14. Gabrilovich D I, Nagaraj S. Myeloid-derived suppressor cells as regulators of the immune system. Nat Rev Immunol. (2009) 9:162-74. doi: 10.1038/nri2506

15. Hsu CY, Lin YC, Chang LY, Huang SK, Huang CH, Yang CK, et al. Therapeutic role of inducible nitric oxide synthase expressing myeloid-derived suppressor cells in acetaminophen-induced murine liver failure. Front Immunol. (2020) 11:574839. doi: 10.3389/fimmu.2020.574839

16. Tengesdal IW, Menon DR, Osborne DG, Neff CP, Powers NE, Gamboni F, et al. Targeting tumor-derived NLRP3 reduces melanoma progression by limiting MDSCs expansion. Proc Natl Acad Sci USA. (2021) 118:e2000915118. doi: $10.1073 /$ pnas. 2000915118 
17. Murray P J, Wynn T A. Protective and pathogenic functions of macrophage subsets. Nat Rev Immunol. (2011) 11:723-37. doi: 10.1038/nri3073

18. Sun YY Li XF, Meng XM, Huang C, Zhang L, Li J. Macrophage phenotype in liver injury and repair. Scand J Immunol. (2017) 85:166-74. doi: $10.1111 /$ sji.12468

19. Shi Y, Su W, Zhang L, Shi C, Zhou J, Wang P, et al. TGR5 regulates macrophage inflammation in nonalcoholic steatohepatitis by modulating NLRP3 inflammasome activation. Front Immunol. (2020) 11:609060. doi: 10.3389/fimmu.2020.609060

20. Kim SJ, Lee SM. NLRP3 inflammasome activation in Dgalactosamine and lipopolysaccharide-induced acute liver failure: role of heme oxygenase-1. Free Radic Biol Med. (2013) 65:997-1004. doi: 10.1016/j.freeradbiomed.2013.08.178

21. Wu X, Dong L, Lin X, Li J. Relevance of the NLRP3 inflammasome in the pathogenesis of chronic liver disease. Front Immunol. (2017) 8:1728. doi: 10.3389/fimmu.2017.01728

22. Coll RC, Robertson AA, Chae JJ, Higgins SC, Muñoz-Planillo R, Inserra $\mathrm{MC}$, et al. A small-molecule inhibitor of the NLRP3 inflammasome for the treatment of inflammatory diseases. Nat Med. (2015) 21:248-55. doi: 10.1038/nm.3806

23. Xu KY, Wu CY, Tong S, Xiong P, Wang SH. The selective Nlrp3 inflammasome inhibitor Mcc950 attenuates lung ischemia-reperfusion injury. Biochem Biophys Res Commun. (2018) 503:3031-7. doi: 10.1016/j.bbrc.2018.08.089

24. Perera AP, Fernando R, Shinde T, Gundamaraju R, Southam B, Sohal SS, et al. MCC950, a specific small molecule inhibitor of NLRP3 inflammasome attenuates colonic inflammation in spontaneous colitis mice. Sci Rep. (2018) 8:8618. doi: 10.1038/s41598-018-26775-w

25. van Hout GP, Bosch L, Ellenbroek GH, de Haan JJ, van Solinge WW, Cooper MA, et al. The selective NLRP3-inflammasome inhibitor MCC950 reduces infarct size and preserves cardiac function in a pig model of myocardial infarction. Eur Heart J. (2017) 38:828-36. doi: 10.1093/eurheartj/ehw247

26. Yu Y, Cheng Y, Pan Q, Zhang YJ, Jia DG, Liu YF. Effect of the selective NLRP3 inflammasome inhibitor mcc950 on transplantation outcome in a pig liver transplantation model with organs from donors after circulatory death preserved by hypothermic machine perfusion. Transplantation. (2019) 103:353-62. doi: 10.1097/TP.0000000000002461

27. Mridha AR, Wree A, Robertson AAB, Yeh MM, Johnson CD, Van Rooyen $\mathrm{DM}$, et al. NLRP3 inflammasome blockade reduces liver inflammation and fibrosis in experimental NASH in mice. J Hepatol. (2017) 66:1037-46. doi: 10.1016/j.jhep.2017.01.022

28. Ostrand-Rosenberg S, Sinha P. Myeloid-derived suppressor cells: linking inflammation and cancer. J Immunol. (2009) 182:4499-506. doi: 10.4049/jimmunol.0802740

29. Nagaraj S, Youn JI, Gabrilovich DI. Reciprocal relationship between myeloidderived suppressor cells and T cells. J Immunol. (2013) 191:17-23. doi: 10.4049/jimmunol.1300654

30. Tengesdal IW, Dinarello A, Powers NE, Burchill MA, Joosten LAB, Marchetti C, et al. Tumor NLRP3-derived IL-1 $\beta$ drives the IL-6/STAT3 axis resulting in sustained MDSC-mediated immunosuppression. Front Immunol. (2021) 12:661323. doi: 10.3389/fimmu.2021.661323
31. Sarra M, Cupi ML, Bernardini R, Ronchetti G, Monteleone I, Ranalli M, et al. IL-25 prevents and cures fulminant hepatitis in mice through a myeloidderived suppressor cell-dependent mechanism. Hepatology. (2013) 58:143650. doi: 10.1002/hep. 26446

32. Xu J, Pei S, Wang Y, Liu J, Qian Y, Huang M, et al. Tpl2 protects against fulminant hepatitis through mobilization of myeloid-derived suppressor cells. Front Immunol. (2019) 10:1980. doi: 10.3389/fimmu.2019. 01980

33. Possamai LA, Thursz MR, Wendon JA, Antoniades CG. Modulation of monocyte/macrophage function: a therapeutic strategy in the treatment of acute liver failure. J Hepatol. (2014) 61:439-45. doi: 10.1016/j.jhep.2014. 03.031

34. Sica A, Invernizzi P, Mantovani A. Macrophage plasticity and polarization in liver homeostasis and pathology. Hepatology. (2014) 59:2034-42. doi: 10.1002/hep. 26754

35. Sica A, Mantovani A. Macrophage plasticity and polarization: in vivo veritas. J Clin Invest. (2012) 122:787-95. doi: 10.1172/JCI59643

36. Wijesundera KK, Izawa T, Tennakoon AH, Murakami H, Golbar HM, KatouIchikawa C, et al. M1- and M2-macrophage polarization in rat liver cirrhosis induced by thioacetamide (TAA), focusing on Ibal and galectin-3. Exp Mol Pathol. (2014) 96:382-92. doi: 10.1016/j.yexmp.2014.04.003

37. Luo Y, Lu J, Ruan W, Guo X, Chen S. MCC 950 attenuated early brain injury by suppressing NLRP3 inflammasome after experimental SAH in rats. Brain Res Bull. (2019) 146:320-6. doi: 10.1016/j.brainresbull.2019.01.027

38. Xu L, Zhang C, Jiang N, He D, Bai Y, Xin Y. Rapamycin combined with MCC950 to treat multiple sclerosis in experimental autoimmune encephalomyelitis. J Cell Biochem. (2019) 120:5160-8. doi: 10.1002/jcb. 27792

39. Ma X, Zheng X, Pan L, Zhang X. NLRP3 inflammasome activation in liver cirrhotic patients. Biochem Biophys Res Commun. (2018) 505:40-4. doi: 10.1016/j.bbrc.2018.09.055

Conflict of Interest: The authors declare that the research was conducted in the absence of any commercial or financial relationships that could be construed as a potential conflict of interest.

Publisher's Note: All claims expressed in this article are solely those of the authors and do not necessarily represent those of their affiliated organizations, or those of the publisher, the editors and the reviewers. Any product that may be evaluated in this article, or claim that may be made by its manufacturer, is not guaranteed or endorsed by the publisher.

Copyright (c) 2021 Yan, Shen, Huang, Lu and Zhang. This is an open-access article distributed under the terms of the Creative Commons Attribution License (CC BY). The use, distribution or reproduction in other forums is permitted, provided the original author(s) and the copyright owner(s) are credited and that the original publication in this journal is cited, in accordance with accepted academic practice. No use, distribution or reproduction is permitted which does not comply with these terms. 